\title{
Bacterias de importancia clínica en respiradores y aires acondicionados de hospitales de San José, Costa Rica.
}

\section{Artículo Original}

María del M. Gamboa, Evelyn Rodríguez, Marianela Rojas.

Laboratorio de Investigación en Bacteriología Anaerobia y Centro de Investigación en Enfermedades Tropicales, Facultad de Microbiología, Universidad de Costa Rica, Costa Rica.

\section{RESUMEN.}

Introducción. El riesgo de adquirir infecciones nosocomiales respiratorias, cuya tasa de mortalidad puede ser hasta del 76\%, aumenta con el uso de respiradores y aires acondicionados, de manera que detectando bacterias de importancia médica en ellos se pretende contribuir a controlar dichas infecciones en hospitales costarricenses.

Material y métodos. En cuatro hospitales, se analizaron 120 muestras de agua acumulada en 79 respiradores y 41 aires acondicionados; cada una se concentró por filtración, se resuspendió y se inoculó en medios de cultivo adecuados. Los aislamientos se identificaron utilizando un sistema semiautomatizado API ${ }^{\circledR}$ y el método de Kirby y Bauer o tiras ATB ${ }^{\circledR}$ para las pruebas de sensibilidad.

Resultados. E1 80\% de los aires acondicionados y $53 \%$ de los respiradores fueron positivos, lo que representa un $63 \%$ de muestras positivas. La mayoría de los aislamientos fueron bacilos Gram negativos (73\%), Pseudomonas y géneros relacionados el grupo más frecuente (47\%), mostrando mayor resistencia antimicrobiana hacia cefalotina (77\%), menor a imipenem (6\%) y una multirresistencia de $64 \%$. Staphylococcus fue el género más frecuente de los cocos Gram positivos (84\%), con la mayor resistencia a rifampicina (86\%), la menor a penicilina (25\%) y una multirresistencia del 50\%; seis cepas (todas coagulasa negativas) fueron resistentes a vancomicina.

Discusión. El alto porcentaje de muestras positivas con bacterias de importancia médica representa un riesgo para los pacientes, especialmente en salas de cuidados intensivos y de cirugía. El predominio de Gram negativos concuerda con su importancia en neumonías nosocomiales; su presencia indica contaminación con el ambiente hospitalario o mala higiene al manipular el equipo. La alta multirresistencia encontrada es preocupante y debe llamar la atención de las autoridades en salud.

(Rev Biomed 2003; 14:143-151)

Palabras clave: Respiradores, aires acondicionados, bacterias Gram negativos, infecciones nosocomiales, resistencia bacteriana.

Solicitud de sobretiros: María del Mar Gamboa, Facultad de Microbiología, Universidad de Costa Rica, Ciudad Universitaria "Rodrigo Facio", Costa Rica, América Central. E-mail: mgamboac@cariari.ucr.ac.cr 
M del M Gamboa, E Rodríguez, M Rojas.

\section{SUMMARY.}

Clinically relevant bacteria in mechanical ventilators and air conditioners in hospitals in San José, Costa Rica.

Introduction. The risk of acquiring respiratory nosocomial infections, whose mortality rate can be as high as $76 \%$, increases with the use of mechanical ventilators and air conditioning. Therefore, our goal was to detect clinically relevant bacteria in them in order to help to control such infections in Costa Rican hospitals.

Material and methods. In four hospitals, 120 samples of accumulated water from 79 mechanical ventilators and 41 air conditioners were analysed; each sample was concentrated by filtration, resuspended, and inoculated in suitable culture media. Isolates were identified using an $\mathrm{API}^{\circledR}$ semiautomated system and the sensitivity tests were done with the Kirby and Bauer method or $\mathrm{ATB}^{\circledR}$ strips.

Results. $80 \%$ of air conditioners and $53 \%$ of the mechanical ventilators were positive for clinically significant bacteria, resulting in $63 \%$ positive samples. Most of the isolates were Gram negative bacilli (73\%), Pseudomonas and related genera were the most frequent $(47 \%)$, showing the highest resistance to cephalothin (77\%), the lowest to imipenem $(6 \%)$, and an antimicrobial multiresistance of $64 \%$. Staphylococcus was the most frequent gender of Gram positive cocci (84\%), with the highest resistance to rifampicin (86\%), the lowest to penicillin $(25 \%)$, and an antimicrobial multiresistance of $50 \%$; six strains (all coagulase negative) were vancomycin resistant.

Discussion. This high percentage of positive samples of clinically significant bacteria represents a risk for patients, especially those in intensive care and surgery units. The predominance of Gram negative bacteria tallies with to its importance in nosocomial pneumonia; its presence indicates contamination of the hospital environment or bad hygiene during the manipulation of the equipment. The high antimicrobial multirresistance found is worrying and should draw health authorities attention to it.

(Rev Biomed 2003; 14:143-151)
Key words: ventilators, air conditioners, Gram negative bacterias, nosocomial infections, bacterial resistance.

\section{INTRODUCCIÓN.}

Tanto en países industrializados como en desarrollo, las infecciones nosocomiales han ido en aumento (1), estimándose que de 5 a $15 \%$ de los pacientes hospitalizados las adquieren (2). En Costa Rica se calcula que anualmente unos 400 pacientes sufren infecciones nosocomiales, para una prevalencia del 9.4\% (3).

Las enfermedades respiratorias representan del 10 al $15 \%$ del total de infecciones nosocomiales (4), de las cuales las neumonías ocurren en el 0.5 al $1.5 \%$ de todos los pacientes hospitalizados y en el 10 al $30 \%$ de aquellos que están con ventilación artificial (5); y la tasa de fatalidad puede ser hasta de $76 \%$ (6). Entre los factores que influyen en el desarrollo de neumonías nosocomiales están la edad avanzada del paciente, el uso de equipo de asistencia respiratoria, ya sea por contaminación o mala manipulación del mismo o por ruptura de las membranas del paciente al colocarlo (7). Los agentes etiológicos más comunes en estas neumonías son Pseudomonas aeruginosa y Staphylococcus aureus $(5,8,9)$.

El uso de respiradores representa un alto riesgo de desarrollar neumonía o traqueobronquitis nosocomiales y, en general, la frecuencia de infección aumenta con la duración de la ventilación mecánica (10). Por otra parte, el empleo de aires acondicionados representa un riesgo, ya que al permitir la diseminación de aerosoles contaminados aumenta la posibilidad de infecciones nosocomiales (11). Algunos patógenos, como Legionella, Pseudomonas o Clostridium, pueden ser distribuidos en el ambiente hospitalario a través de sistemas de aire acondicionado defectuosos (12).

Los pacientes admitidos a las unidades de cuidados intensivos (UCI) tienen un mayor riesgo de adquirir infecciones nosocomiales que aquellos pacientes de otras áreas del hospital (13). Así Eggimann y Pittet (2) refieren que de 25 a $33 \%$ de los pacientes admitidos en UCI las presentan;

\section{Revista Biomédica}


Bacterias en respiradores y aires acondicionados de hospitales.

específicamente, la neumonía asociada a ventiladores ocurre en 8 a $28 \%$ de los pacientes con ventilación mecánica (6).

La prevención de las neumonías nosocomiales es uno de los pasos más importantes para reducir los costos de hospitalización, de manera que este trabajo pretende contribuir al control de infecciones nosocomiales en hospitales de San José de Costa Rica, mediante la determinación de la presencia de bacterias de importancia clínica en sistemas de ventilación asistida y aires acondicionados de unidades de cuidados intensivos y otros servicios donde se encuentren pacientes con mayor riesgo de adquirir infecciones respiratorias.

\section{MATERIAL Y MÉTODOS.}

Se analizaron 120 muestras de agua acumulada en respiradores (79) y aires acondicionados (41), provenientes de cuatro hospitales de San José, desglosadas así: 29 del Hospital A, 26 del B, 37 del C y 28 del D (Cuadro 1). El agua acumulada en cada equipo se colocó en bolsas estériles que fueron transportadas en frío y analizadas en menos de dos horas. Cada muestra se concentró utilizando un filtro de policarbonato con poros de $0,22 \mathrm{~mm}$ que se colocó en un frasco con $10 \mathrm{ml}$ de la muestra original y se agitó con Vórtex por 30 seg. Se inoculó, por esparcimiento, $0,1 \mathrm{ml}$ de esta suspensión en agares sangre, chocolate, manitol sal, MacConkey y cetrimida. Todas las placas se incubaron a $37^{\circ} \mathrm{C}$, excepto las de agar Cetrimida que fueron a $30^{\circ} \mathrm{C}$, por un máximo de 7 días; las de agar sangre y chocolate se incubaron en atmósfera aumentada de dióxido de carbono.

A cada morfotipo colonial se le hizo tinción de Gram y pruebas de oxidasa y catalasa. Para su identificación, de acuerdo con su morfología, se utilizó alguno de los sistemas semiautomatizados API (Biomérieux ${ }^{\circledR}$ ), siguiendo las recomendaciones del fabricante. Para la prueba de sensibilidad a los antibióticos de los bacilos Gram negativos se utilizó el método de Kirby y Bauer de acuerdo con las recomendaciones del Comité Nacional para Estándares del Laboratorio Clínico (NCCLS, por sus siglas en inglés, 14), para los estreptococos ATB STREP 5 (Biomérieux ${ }^{\circledR}$ ) y para los estafilococos ATB STAPH 5 (Biomérieux $®)$. Para determinar la sensibilidad de los estafilococos a la oxacilina se siguió la técnica de Kirby y Bauer modificada (15).

\section{Cuadro 1}

Respiradores y aires acondicionados positivos por bacterias de importancia clínica en cuatro hospitales de San José, Costa Rica.

\begin{tabular}{lcrrrr}
\hline $\begin{array}{c}\text { Hospital Aires acondicion. } \\
\text { Muestras+/ } \\
\text { total }\end{array}$ & $\%$ & $\begin{array}{c}\text { Respiradores } \\
\text { Muestras+/ } \\
\text { total }\end{array}$ & $\begin{array}{c}\text { Total } \\
\text { Muestras } \\
\text { \% }\end{array}$ \\
\hline $\mathrm{A}$ & $6 / 9$ & 67 & $14 / 20$ & 70 & 69 \\
$\mathrm{~B}$ & $9 / 9$ & 100 & $7 / 28$ & 25 & 43 \\
$\mathrm{C}$ & $9 / 9$ & 100 & $13 / 17$ & 76 & 85 \\
$\mathrm{D}$ & $9 / 14$ & 64 & $8 / 14$ & 57 & 61 \\
Total & $33 / 41$ & 80 & $42 / 79$ & 53 & 63 \\
\hline
\end{tabular}

\section{RESULTADOS.}

En 75 de las 120 muestras analizadas (63\%) se aislaron bacterias de importancia clínica, desde $43 \%$ hasta $85 \%$ según el hospital. En términos generales y de acuerdo con el tipo de muestra, el $80 \%$ de los aires acondicionados y el $53 \%$ de los respiradores fueron positivos. Es importante destacar que dos de los hospitales (B y C) tuvieron todos los aires acondicionados con resultados positivos; por otra parte, en uno (B) sólo el $25 \%$ de los respiradores fue positivo y en otro (C) el $76 \%$ de los respiradores fue positivo (cuadro 1).

Se aislaron 143 bacterias, de las cuales 105 (73\%) eran bacilos Gram negativos y 39 (27\%) eran cocos Gram positivos. Este predominio de los bacilos Gram negativos se dio en todos los hospitales: $55 \%$ en el hospital A, $76 \%$ en el B, $71 \%$ en el C y $89 \%$ en el D. Al identificarlas se encontró que la mayoría eran Pseudomonas y géneros relacionados (47\%), seguido de las enterobacterias (16\%) y del grupo de Acinetobacter (13\%) (cuadro 2). En todos los hospitales Pseudomonas fue el bacilo Gram negativo más aislado, excepto en el B, en el cual Flavobacterium y Acinetobacter fueron los más 
M del M Gamboa, E Rodríguez, M Rojas.

Cuadro 2

Frecuencia de bacilos Gram negativos de importancia clínica aislados en cuatro hospitales de San José, Costa Rica.

\begin{tabular}{|c|c|c|c|c|c|}
\hline \multirow[b]{2}{*}{ Bacterias } & \multicolumn{4}{|c|}{ Hospitales* } & \multirow[b]{2}{*}{$\begin{array}{c}\text { Total } \\
\%\end{array}$} \\
\hline & $\begin{array}{l}\text { A } \\
\%\end{array}$ & $\begin{array}{l}\text { B } \\
\%\end{array}$ & $\begin{array}{l}\mathrm{C} \\
\%\end{array}$ & $\begin{array}{l}\mathrm{D} \\
\%\end{array}$ & \\
\hline Pseudomonas ${ }^{\dagger}$ & 47 & 22 & 63 & 59 & 47 \\
\hline Enterobacteriaceae & 35 & 22 & 4 & 9 & 16 \\
\hline Acinetobacter & 12 & 25 & 0 & 13 & 13 \\
\hline Flavobacterium & 6 & 25 & 8 & 0 & 10 \\
\hline Moraxella & 0 & 6 & 4 & 19 & 9 \\
\hline Pasteurella & 0 & 0 & 8 & 0 & 2 \\
\hline Alcaligenes & 0 & 0 & 4 & 0 & 1 \\
\hline Flavimonas & 0 & 0 & 8 & 0 & 2 \\
\hline
\end{tabular}

* $\mathrm{N}^{\mathrm{o}}$ de aislamientos por hospital: 17en A, 32 en B, 24 en C y 32 en D.

$\dagger$ incluye Ralstonia, Burkholderia, Brevundimonas y Stenotrophomonas.

* incluye Chryseobacterium y Weeksella.

frecuentes (25\% cada uno) (cuadro 2).

Staphylococcus fue el género de cocos Gram positivos que se aisló con más frecuencia (77\%), situación que se mantuvo en todos los hospitales y que osciló desde 50\% hasta 100\% (cuadro 3); las dos especies más frecuentes fueron $S$. haemolyticus y S. hominis. Por otro lado, los géneros Micrococcus y Aerocococcus sólo se aislaron en uno de los hospitales (cuadro 3).

Respecto a la sensibilidad a los antimicrobianos

Cuadro 3

Frecuencia de cocos Gram positivos de importancia clínica aislados en hospitales de San José, Costa Rica.

\begin{tabular}{lrrrrc}
\hline \multirow{2}{*}{ Bacterias } & \multicolumn{5}{c}{ Hospitales* } \\
& A & B & C & D & Total \\
& $\%$ & $\%$ & $\%$ & $\%$ & $\%$ \\
\hline Staphylococcus & 79 & 64 & 100 & 50 & 77 \\
Streptococcus & 21 & 0 & 0 & 50 & 13 \\
Micrococcus & 0 & 27 & 0 & 0 & 8 \\
Aerococcus & 0 & 9 & 0 & 0 & 2 \\
\hline
\end{tabular}

$* \mathrm{~N}^{\mathrm{o}}$ de aislamientos por hospital: 14 en $\mathrm{A}, 11$ en $\mathrm{B}, 10$ en $\mathrm{C}$ y 4 en D.

\section{Revista Biomédica}

\section{Cuadro 4}

Porcentaje de sensibilidad a los antimicrobianos de bacilos Gram negativos aislados en cuatro hospitales de San José, Costa Rica.

\begin{tabular}{lccccc}
\hline & \multicolumn{5}{c}{ Hospitales* } \\
Antibiótico & A & B & C & D & Total \\
\hline Imipenem & 93 & 88 & 100 & 94 & 94 \\
Ciprofloxacina & 87 & 63 & 92 & 84 & 84 \\
Tetraciclina & 60 & 63 & 92 & 81 & 81 \\
Piperacilina & 67 & 46 & 100 & 73 & 73 \\
Cloranfenicol & 53 & 67 & 83 & 67 & 67 \\
Trimetro.Sulfa & 60 & 38 & 67 & 66 & 66 \\
Gentamicina & 87 & 58 & 67 & 63 & 63 \\
Cefuroxime & 47 & 21 & 71 & 59 & 59 \\
Tobramicina & 73 & 58 & 50 & 53 & 53 \\
Amoxacilina & 13 & 25 & 50 & 29 & 29 \\
Cefalotina & 27 & 25 & 8 & 23 & 23 \\
\hline
\end{tabular}

$* \mathrm{~N}^{\mathrm{o}}$ de aislamientos por hospital: 15 en A, 24 en $\mathrm{B}, 24$ en $\mathrm{C}$ y 32 en D.

Cuadro 5

Porcentaje de sensibilidad a antimicrobianos de Staphylococcus aislados de cuatro hospitales de San José, Costa Rica.

\begin{tabular}{lccccc}
\hline \multicolumn{5}{c}{ Hospitales* } \\
Antibiótico & A & B & C & D & Total \\
\hline Rifampicina & 73 & 80 & 100 & 100 & 86 \\
Oxacilina & 91 & 40 & 90 & 100 & 82 \\
Teicoplanina & 73 & 80 & 90 & 100 & 82 \\
Tetraciclina & 73 & 60 & 100 & 100 & 82 \\
Nitrofurantoína & 73 & 80 & 80 & 100 & 79 \\
Ampicil. sulbactam & 73 & 40 & 100 & 100 & 79 \\
Vancomicina & 73 & 40 & 90 & 100 & 79 \\
Ciprofloxacina & 73 & 20 & 90 & 100 & 71 \\
Netilmicina & 73 & 40 & 80 & 100 & 71 \\
Cotrimazole & 55 & 20 & 100 & 100 & 68 \\
Cefalotina & 63 & 20 & 80 & 100 & 64 \\
Gentamicina & 55 & 20 & 90 & 100 & 64 \\
Pefloxacina & 73 & 0 & 70 & 100 & 61 \\
Clindamicina & 45 & 20 & 80 & 100 & 57 \\
Eritromicina & 27 & 20 & 70 & 50 & 43 \\
Penicilina & 0 & 0 & 60 & 50 & 25 \\
\hline
\end{tabular}

* $\mathrm{N}^{\mathrm{o}}$ de aislamientos por hospital: 11 en A, 5 en $\mathrm{B}, 10$ en $\mathrm{C}$ y 2 en D. 
Bacterias en respiradores y aires acondicionados de hospitales.

de los bacilos Gram negativos y considerando todos los hospitales, el de mayor valor fue al imipenem (94\%), seguida de la ciprofloxacina (84\%, cuadro 4). La mayor resistencia fue a la amoxacilina y a la cefalotina, ya que sólo 29\% y 23\% de las cepas, respectivamente, fue sensible a estos antibióticos. El desglose de sensibilidad de las cepas de bacilos Gram negativos en los diferentes hospitales se muestra en el cuadro 5, observándose una sensibilidad antimicrobiana similar para ciertas drogas como el imipenem (88 a 100\% de las cepas sensibles) y diferentes para otras, como la piperacilina (de 46 a $100 \%$ de cepas sensibles) o el cefuroxime (de 21 a $71 \%$ de cepas sensibles).

Referente a la sensibilidad del grupo de los Staphylococcus, la rifampicina fue el antibiótico al que la mayoría de las cepas fue sensible (86\%); la menor sensibilidad fue hacia penicilina (25\%) (cuadro 5). A pesar de que el $82 \%$ de las cepas fue sensible a la oxacilina, es importante hacer notar que en uno de los hospitales (B) sólo el 40\% de las cepas lo fue (cuadro 5). Aunque se encontraron cepas resistentes a vancomicina en tres de los cuatro hospitales (cuadro 5), todas eran Staphylococcus coagulasa negativas. En los hospitales A y B ninguna cepa fue sensible a la penicilina y en el B ninguna a la pefloxacina. En el D se observó que las cepas aisladas eran sensibles a todos los antibióticos evaluados, excepto penicilina y eritromicina; sin embargo, la sensibilidad antimicrobiana hacia estos dos antibióticos fue mayor que el valor promedio de todos los hospitales.

Se analizó la resistencia bacteriana múltiple considerando las cepas con resistencia a tres o más antibióticos, encontrándose que, en todos los hospitales, el $64 \%$ de los bacilos Gram negativos y el $50 \%$ de los cocos Gram positivos la presentó; el 16\% de los Gran negativos fue resistente al menos a seis antibióticos, mientras que el $22 \%$ de los cocos fue resistente al menos a 10, situación más acentuada en el hospital B, pues el $75 \%$ de los cocos de éste fue resistente a 10 antibióticos. Tomando en cuenta todas las cepas aisladas en cada hospital se observó la mayor multirresistencia en el B (84\%), seguida de $65 \%$ en el C, $62 \%$ en el A y $53 \%$ en el D.

\section{DISCUSIÓN.}

La posibilidad de transmisión vía aérea, directa o indirecta, de bacterias patógenas hospitalarias ha sido subestimada (12). Por ejemplo, la contaminación de la herida en una cirugía con frecuencia se debe a bacterias que caen directamente del ambiente, lo cual hace necesario controlar la fuente de contaminación $(16,17)$. El aislamiento de bacterias de importancia clínica en un alto porcentaje de las muestras de aires acondicionados y respiradores $(63 \%)$ puede representar un riesgo para los pacientes, tomando en cuenta que muchos de los sitios de muestreo fueron salas de cuidados intensivos y salas de operación en donde los pacientes tienen mayor riesgo de adquirir infecciones nosocomiales que los pacientes de otras unidades $(7,13)$.

Al analizar la distribución de las muestras positivas por hospital se observó que no fue homogénea: en uno de los hospitales (B) el 85\% de las muestras lo fue, mientras que en otro hospital (C) sólo el $43 \%$. Esto puede atribuirse a condiciones particulares de cada hospital, localización geográfica, estado de las edificaciones, condiciones de higiene, etc.

El aislamiento de bacterias de importancia clínica a partir de un alto porcentaje de los aires acondicionados $(80 \%)$ representa un riesgo de infección para los pacientes (11). En dos de los hospitales (B y C) todos los aires acondicionados resultaron positivos, lo que pudo deberse al esquema de mantenimiento de cada hospital y a la frecuencia con que se cambien los filtros.

Aun cuando los respiradores presentaron un menor grado de contaminación con bacterias de importancia clínica (53\%) que los aires acondicionados, hay que recalcar que el empleo de dispositivos invasivos es un importante factor de riesgo para infecciones. El uso de ventiladores se ha relacionado con una mayor incidencia de neumonías nosocomiales $(7,18)$ e incluso, a partir de equipos de ventilación asistida se ha descrito el aislamiento de agentes causales, tales como Stenotrophomonas maltophilia (19). Las diferencias en cuanto al porcentaje de respiradores positivos en los diferentes 


\section{M del M Gamboa, E Rodríguez, M Rojas.}

hospitales pueden deberse al tipo de respirador utilizado en cada uno y a la manipulación del equipo. El papel fundamental que pueden jugar los respiradores y los aires acondicionados en la diseminación de microorganismos por vía aérea resalta la importancia del alto grado de contaminación detectado, siendo la dispersión aérea una manera de adquirir infecciones en los hospitales (20).

Según datos de la Organización Mundial de la Salud sobre neumonías nosocomiales, $P$. aeruginosa es el patógeno más frecuente a nivel mundial y el segundo más común, después de $S$. aureus, en los Estados Unidos (7); Barcenilla (13) y Chastre y Fagon (6) los señalan como los agentes etiológicos más importantes en las neumonías asociadas a sistemas invasivos de ventilación mecánica. En Costa Rica en un estudio previo sobre infecciones nosocomiales (3), se encontró que los bacilos Gram negativos fueron las bacterias más frecuentes, particularmente $P$. aeruginosa, mientras que Staphylococcus fue el género de Gram positivos más aislado.

El ambiente hospitalario, rico en agua y nutrientes, brinda un nicho a los bacilos Gram negativos que les ha permitido convertirse en importantes agentes de infección nosocomial (7). En este estudio los bacilos Gram negativos predominaron en todos los hospitales desde un 55\% hasta un $89 \%$. Numerosos estudios refieren que las neumonías nosocomiales son causadas más frecuentemente por bacilos Gram negativos. Así, en Norteamérica son responsables del 60\% (20), en Taiwán del 53\% (21) $\mathrm{y}$ en Brasil del 54\% (22).

Las bacterias Gram negativas que se aislaron en mayor proporción fueron Pseudomonas y bacterias que antes pertenecían a este género como Ralstonia, Burkholderia, Brevundimonas y Stenotrophomonas, representando en conjunto un $47 \%$ de las bacterias Gram negativas (cuadro 2). Éstas son habitantes normales del ambiente y no se consideran parte de la flora normal humana (8), por lo que su presencia en los respiradores es indicadora de contaminación y representa un riesgo, puesto que son capaces de sobrevivir en equipo médico y en desinfectantes (7). Es importante recalcar que algunas de estas especies, como Burkholderia cepacia, $P$. aeruginosa y S. maltophilia, son agentes etiológicos de neumonías nosocomiales $(8,19)$. De las cepas de $P$. aeruginosa sólo una se aisló a partir de un respirador, las demás a partir de aires acondicionados; pero tomando en cuenta el potencial patógeno de esta bacteria esto representa un riesgo para la salud de pacientes que podrían estar en condición crítica.

El aislamiento de especies de Enterobacteriaceae como Escherichia coli y Klebsiella pneumoniae, el segundo grupo más frecuente (cuadro 2), es preocupante ya que indica una mala higiene al manipular el equipo o bien que se esté utilizando agua contaminada. Otros géneros de Gram negativos que se aislaron, como Acinetobacter, Moraxella y Flavobacterium, también son agentes responsables de infecciones intrahospitalarias (8). Así, Costa et al. (22) encontraron que $A$. baumannii fue responsable del 29\% de las neumonías nosocomiales.

Como se anotó antes, Staphylococcus fue el género de bacterias Gram positivas más importante en infecciones nosocomiales en Costa Rica y lo fue también en este estudio (cuadro 3). Mientras que $S$. aureus ha sido reconocido como una causa importante de morbilidad y mortalidad, el papel de las especies coagulasa negativa como causantes de este tipo de infecciones se ha documentado sólo en los últimos 20 años (23). S. haemolyticus y S. hominis, las dos especies de estafilococos que más frecuentemente se aislaron en este trabajo, aunque son habitantes normales de la piel humana, con frecuencia se asocian con infecciones en humanos (23).

La aparición de resistencia a los antimicrobianos tiene un papel crítico en las infecciones nosocomiales. Cuando los pacientes con ventilación asistida desarrollan una infección, generalmente están involucrados microorganismos con alta resistencia antimicrobiana (10). Algunos autores señalan que no se deberían usar antibióticos con un alto potencial de resistencia para tratar las neumonías nosocomiales, e incluyen en este grupo, entre otros, al imipenem y a la ciprofloxacina (24). En nuestro estudio, las cepas de bacilos Gram negativos encontradas y que podrían

\section{Revista Biomédica}




\section{Bacterias en respiradores y aires acondicionados de hospitales.}

llegar a causar neumonías por fuentes exógenas, mostraron una alta sensibilidad al impenem (94\%), quizá debido a un uso muy racional de esta droga en los hospitales. Sin embargo, en el hospital B los bacilos Gram negativos presentaron un mayor porcentaje de resistencia hacia el imipem (12\%) y las Pseudomonas aún mayor (29\%). Estos datos están acordes con el comportamiento general de las cepas de este hospital hacia las otras drogas: los porcentajes de sensibilidad hacia ocho antibióticos fueron menores al promedio general de todos los hospitales.

Aunque la sensibilidad a la ciprofloxacina, considerando todos los hospitales, fue de $84 \%$, en uno de los hospitales sólo el $63 \%$ de las cepas lo fue (cuadro 4). Aunque Costa y col. (22) señalan que la ciprofloxacina podría usarse como la mejor alternativa para la terapia inicial empírica de las neumonías, nuestros datos sugieren una evaluación más minuciosa para instaurar dicha práctica y están más acordes con Cunha (24) quien señala la necesidad de un uso más cuidadoso al respecto.

La mayor resistencia bacteriana hacia la cefalotina $(77 \%)$ y a la amoxicilina $(71 \%)$ podría explicarse por el amplio uso de dichas drogas en nuestro medio; tanto las cefalosporinas de primera generación (incluyendo cefalotina) como la amoxicilina, están dentro de las drogas de mayor uso en los hospitales al momento del estudio.

Al analizar los datos de los cocos Gram positivos, se observó que la mayoría de los Staphylococcus fueron sensibles a la rifampicina (86\%), antimicrobiano de amplio espectro, efectivo contra cocos Gram positivos e incluso cepas resistentes a meticilina (25). En nuestro estudio, el porcentaje de sensibilidad a la oxacilina (meticilina), considerando todas las cepas, fue de $82 \%$, pero es muy importante señalar nuevamente las diferencias en cada hospital: desde $100 \%$ sensibles en el hospital D hasta $40 \%$ sensibles en el B (cuadro 5). La alta resistencia en este hospital es preocupante, pues $S$. aureus, incluyendo cepas meticilina resistentes, continúa siendo uno de los agentes más frecuentes en neumonías nosocomiales (26). Las cepas de Staphylococcus resistentes a vancomicina correspondieron a especies coagulasa negativas, situación que ya ha sido informada previamente (27).

La baja sensibilidad hacia la penicilina (25\%) de las cepas de todos los hospitales no es de extrañar, pues desde 1980 y cada vez con más frecuencia, se han aislado cepas productoras de beta lactamasas (28). La resistencia múltiple encontrada en este mismo grupo $(50 \%)$, aunque alarmante, era de esperar, pues la presencia continua de diferentes antibióticos en el ambiente hospitalario ejerce la presión de selección para favorecer a cepas multirresistentes. Los bacilos Gram negativos tampoco han permanecido ajenos a esta situación y no menos preocupante fue el hallazgo de cepas resistentes hasta a nueve antibióticos simultáneamente. La mayor resistencia de las cepas en uno de los hospitales se vio reflejada en la alta frecuencia de multirresistencia, pues el $75 \%$ de los Staphylococcus de ese centro hospitalario fue resistente a 10 antibióticos.

$\mathrm{Al}$ analizar el comportamiento de las cepas de bacilos Gram negativos en cada hospital se encontró un patrón de sensibilidad mayor en el hospital C y menor en el B, puesto que las cepas del primero sólo tuvieron porcentajes de sensibilidad menores al promedio de todos los hospitales hacia dos de las 11 drogas analizadas (tobramicina y cefalotina), en tanto que las cepas del B fueron menos sensibles que el promedio general hacia ocho de las 11 drogas (imipenem, tetraciclina, ciprofloxacina, trimetoprimsulfa, cefuroxime, piperacilina, gentamicina y amoxicilina, cuadro 4). En lo que respecta a Staphylococcus en cada hospital, el patrón de sensibilidad bacteriana hacia los 16 antimicrobianos evaluados fue variado, pues en dos de los hospitales (A y B) las cepas fueron mucho más resistentes que en los otros dos, ya que mostraron una sensibilidad inferior al promedio hacia 12 y 15 drogas, respectivamente.

Las variaciones en cada hospital en cuanto a la sensibilidad de los cocos Gram positivos hacia los antimicrobianos evaluados también fue manifiesta, siendo muy marcada hacia la la gentamicina: $100 \%$ de las cepas del hospital D sensibles, pero sólo 20\% en el B. La gentamicina ha sido incluida dentro de las 


\section{M del M Gamboa, E Rodríguez, M Rojas.}

drogas de alto potencial de resistencia (24) por lo que cada vez es más frecuente la aparición de cepas de Staphylococcus resistentes a la gentamicina (29), hallazgo evidente en uno de los hospitales.

Las diferencias encontradas en el comportamiento hacia los antibacterianos en los diferentes hospitales refuerzan la necesidad de que se realicen evaluaciones periódicas tendientes a conocer las cepas bacterianas que permanecen en cada hospital y su perfil de sensibilidad. Esto es especialmente válido en servicios como las unidades de cuidado intensivo, pues de acuerdo con Luna y colaboradores (30) más del $80 \%$ de los pacientes de la UCI reciben tratamiento antimicrobiano profiláctico, que debe ser racional y cuidadoso especialmente en aquellos pacientes que requieren ventiladores mecánicos (31); además, las neumonías nosocomiales en pacientes, ya sea con ventilación asistida o no, requieren diferentes tratamientos antimicrobianos empíricos (32).

La prevención de neumonías nosocomiales es el paso más importante para reducir los costos de hospitalización y para lograrlo es necesario adoptar estrategias preventivas que incluyan medidas relacionadas con el mantenimiento del equipo mecánico de ventilación (33) así como de los aires acondicionados. El ambiente hospitalario se volverá más importante a medida que aumenten el número de pacientes inmunosupresos y el número de infecciones nosocomiales debidas a organismos resistentes.

\section{AGRADECIMIENTOS.}

Agradecemos a Pablo Vargas y a Martín Quesada por su ayuda técnica, y a la Vicerrectoría de Investigación de la Universidad de Costa Rica por el financiamiento ( $N^{\circ} 430-97-$ 257).

\section{REFERENCIAS.}

1.- Hasan R, Babar SI. Nosocomial and ventilator-associated pneumonias: developing country perspective. Curr Opin Pulm Med 2002; 8:188-94.

2.- Eggimann P, Pittet D. Infection control in ICU. Chest 2001; 120:2059-93.

3.- Caja Costarricense del Seguro Social. Primera Prevalencia Nacional. Comisión Gerencial de Prevención y Control de
Infección Intrahospitalaria. San José, Costa Rica, 1997.

4.- Ferrer R, Ioanas M, Agusti C, Torres A. Impact of BAL on the diagnosis and treatment of nosocomial pneumonia in ICU patients. Monaldi Arch Chest Dis 2001; 56:221-6.

5.- Fagon JY. Epidemiology and antibiotic therapy in nosocomial pneumonia. Rev Pneumol Clin 2001; 57:132-8.

6.- Chastre J, Fagon JY. Ventilator-associated pneumonia. Am J Respir Crit Care Med 2002; 165:867-903.

7.- Dembry LM, Zervos MJ, Hierholzer WJ. Nosocomial bacterial infections. En: Evans AS, Brachman PS, editores. Bacterial Infections of Humans. Epidemiology and Control. 3rd. ed. New York: Plenum Publishing Co.; 1998. p. 501-28.

8.- Forbes BA, Sahm DF, Weissfeld SA. Bailey \& Scott's Diagnostic Microbiology. 10th. ed. St. Louis: Mosby; 1998. p. 448-61.

9.- Akova M. Hospital-acquired respiratory tract infections: clinical experience with beta-lactam/beta-lactamase inhibitors. Int J Clin Pract Suppl 2002; 125: 19-27.

10.- Ahmed QA, Niederman MS. Respiratory infection in the chronically critically ill patient. Ventilator-associated pneumonia and tracheobronchitis. Clin Chest Med 2001; 22:71-85.

11.- McDonald LC, Walker M, Carson L, Arduino M, Aguero SM, Gomez P, et al. Outbreak of Acinetobacter spp. Bloodstream infections in a nursery associated with contaminated aerosols and air conditioners. Pediatr Infect Dis J 1998; 17:716-22.

12.- Schaal KP. Medical and microbiological problems arising from airborne infection in hospitals. J Hosp Infect 1991; 18 (Suppl A): 451-9.

13.- Barcenilla F, Gasco E, Rello J, Alvarez -Rocha L. Drugs Aging 2001; 18:189-200.

14.- Jorgensen JH, Turnidge JD, Washington JA. Antibacterial susceptibility tests: Dilution and disk diffusion methods. En: Murray PR. Editor, Manual of Clinical Microbiology. 7th. ed. Washington: ASM Press; 1999. p. 1526-43.

15.- Leitch C, Boonlayangoor S. Tests to detect oxacillin (Methicillin)-resistant staphylococci with an oxacillin screen plate. En: Isenberg HD, editor. Clinical Microbiological Procedures. Washington: ASM Press; 1992. Vol. 1: p. 5.5.15.5.7.

\section{Revista Biomédica}




\section{Bacterias en respiradores y aires acondicionados de hospitales.}

16.- Duhaime AC, Bonner K, McGowan KL, Schut L, Sutton LN, Plotkin S. Distribution of bacteria en the operating room and its relation to ventricular shunt infections: a prospective study. Childs Nerv Syst 1991; 4: 211-4

17.- Ritter MA. Operating room environment. Clin Orthop 1999; 369: 103-9.

18.- Martínez-Aguilar G, Anaya-Arriaga MC, Ávila-Figueroa C. Incidencia de bacteremia y neumonía nosocomial en una unidad de pediatría. Salud Pública Mex 2001; 43: 515-23.

19.- Rogues AM, Maugein J, Allery A, Fleureau C, Boulestreau H, Surcin S, et al. Electronic ventilator temperature sensors as a potential source of respiratory tract colonisation with Stenotrophomonas maltophilia. J Hosp Infect 2001; 49: 289-92.

20.- Herwaldt LA, Wenzel R. Dynamics of hospital-acquired infection. En: Murray PR, editor. Manual of Clinical Microbiology. 6th. ed. Washington: ASM Press; 1995. p. 169-81.

21.- Hsueh PR, Chen ML, Sun CC, Chen WH, Pan HJ, Yang LS, et al. Antimicrobial drug resistance in pathogens causing nosocomial infections at a university hospital in Taiwan, 19811999. Emerg Infect Dis 2002; 8:63-8.

22.- Costa SF, Newbaer M, Santos CR, Basso M, Soares I, Levin AS. Nosocomial pneumonia: importance of recognition of aetiological agents to define an appropiate initial empirical therapy. Int J Antimicob Agents 2001; 17:147-50.

23.- Kloos WE, Bannerman TL. Staphylococcus and Micrococcus. En: Murray PR, editor. Manual of Clinical Microbiology. 7th. ed. Washington: ASM Press; 1999. p. 264-282.

24.- Cunha BA. Nosocomial pneumonia. Diagnostic and therapeutic considerations. Med Clin North Am 2001; 85:79114.

25.- Turnidge JD, Jorgensen JH. Antimicrobial susceptibility testing: General considerations. En: Manual of Clinical Microbiology. 7th. ed. Washington: ASM Press; 1999. p.146973.

26.- Marrie TJ. Pneumonia in the long-term-care facility. Infect Control Hosp Epidemiol 2002; 23:159-64.

27.- Projan SJ. Antibiotic resistance in the staphylococci. En: Fischetti VA, Novick RP, Ferretti JJ, Portnoy DA, Rood JI, editores. Gram-Positive Pathogens. Washington: ASM Press;
2000. p. 463-70.

28.- Bodi M, Ardanuy C, Rello J. Impact of Gram-positive resistance on outcome of nosocomial pneumonia. Crit Care Med 2001; 29:82-6.

29.- Witte W, Braulke C, Cuny C, Heuck D, Kresken M. Changing pattern of antibiotic resistance in methicillinresistant Staphylococcus aureus from German Hospitals. Infect Control Hosp Epidemiol 2001; 22:683-6.

30.- Luna CM, Gherardi C, Famiglietti A, Vay C. Bacterial resistance and antimicrobial therapy in respiratory medicine and intensive care. Medicina (B Aires) 2001; 61 (5 Pt 1): 603613.

31.- Sintchenko V, Iredell JR, Gilbert GL. Antibiotic therapy of ventilator-associated pneumonia-a reappraisal of rationale in the era of bacterial resistance. Int J Antimicrob Agents 2001; 18: 223-9.

32.- Ewig S, Walger P, Vetter H. Nosocomial pneumonia. Ther Umsch 2001; 58:609-13.

33.- Ferrer R, Artigas A. Clinical review: non-antibiotic strategies for preventing ventilator-associated pneumonia. Crit Care 2002; 6:45-51. 